\title{
THE HE-STRONG STAR HD 96446: OBLIQUE ROTATOR OR PULSATING MAGNETIC VARIABLE?
}

\author{
G. MATHYS \\ ESO, Casilla 19001, Santiago 19, Chile
}

HD 96446 is a B2p He-strong star, in which a large-scale organized magnetic field is present. From spectra recorded in both circular polarizations, various moments of this magnetic field have been repeatedly determined, among which the mean longitudinal magnetic field and the crossover. The mean longitudinal magnetic field $\left\langle H_{z}\right\rangle$ is the line-intensity weighted average over the visible stellar hemisphere of the line-of-sight component of the magnetic vector. The crossover, $v_{\mathrm{e}} \sin i\left\langle x H_{z}\right\rangle$, is the product of the projected equatorial velocity, $v_{\mathrm{e}} \sin i$, and of the mean asymmetry of the longitudinal magnetic field, $\left\langle x H_{z}\right\rangle$. The latter is the line-intensity weighted first-order moment about the plane defined by the line of sight and the stellar rotation axis of the component of the magnetic field along the line of sight.

Matthews \& Bohlender (1991) have shown that the longitudinal magnetic field and the brightness of HD 96446 vary with the same period $P=0.85137$. In agreement with the oblique rotator model (ORM), which accounts well for the observed magnetic and photometric variations of most Ap and Bp stars, they interpret this period as the period of rotation of the star.

The variations of $\left\langle H_{z}\right\rangle$ with rotation phase $\phi$ in HD 96446 are well approximated by a sinusoid, $\left\langle H_{z}\right\rangle=H_{0}+H_{1} \cos \left[2 \pi\left(\phi+\phi_{1}\right)\right]$, with $H_{0}=$ $-(986 \pm 53) \mathrm{G}, H_{1}=(242 \pm 73) \mathrm{G}$, and $\phi_{1}=0.617 \pm 0.049$. Such closely sinusoidal variations are typical of many Ap and Bp stars. Interpreted within the frame of the ORM, they suggest that the magnetic fields of most Ap and $\mathrm{Bp}$ stars are dominated by a single dipolar component, located close to the stellar centre.

For a stellar magnetic field consisting of a single dipole, whose centre coincides with the centre of the star, and whose axis makes an angle $\beta$ with respect to the stellar rotation axis, approximate analytic expressions of the variations of $\left\langle H_{z}\right\rangle$ and of $v_{e} \sin i\left\langle x H_{z}\right\rangle$ with rotation phase can be derived. Both quantities vary sinusoidally, in phase quadrature with respect to each other. For non-saturated spectral lines (an excellent approximation in HD 96446) and a linear limb darkening law of the form $1-u+u \cos \theta$ (with usual notations; $0 \leq u \leq 1$ ), one gets the following relation between the maximum $\left\langle H_{z}\right\rangle^{+}$and the minimum $\left\langle H_{z}\right\rangle^{-}$of the mean longitudinal field, and the extrema $v_{\mathrm{e}} \sin i\left\langle x H_{z}\right\rangle^{+}$and $v_{\mathrm{e}} \sin i\left\langle x H_{z}\right\rangle^{-}$of the crosover:

$$
v_{\mathrm{e}} \sin i\left(\left\langle x H_{z}\right\rangle^{+}-\left\langle x H_{z}\right\rangle^{-}\right) /\left(\left\langle H_{z}\right\rangle^{+}-\left\langle H_{z}\right\rangle^{-}\right)=1.5 v_{\mathrm{e}}(8-3 u) /(15+u) \text {. }
$$


In HD 96446, no definite detection of the crossover was achieved. From this, we derive: $v_{\mathrm{e}} \sin i\left[\left\langle x H_{z}\right\rangle^{+}-\left\langle x H_{z}\right\rangle^{-}\right] \leq 16.3 \mathrm{kG} \mathrm{km} \mathrm{s}^{-1}$. This is a very conservative $2 \sigma$ upper limit, computed from the largest $\sigma$ of all the determinations of the crossover performed for this star. On the other hand, $\left\langle H_{z}\right\rangle^{+}-\left\langle H_{z}\right\rangle^{-}=2 H_{1}$. Thus, one can derive an upper limit of the equatorial velocity $v_{e}$, hence, as the rotation period is known, of the stellar radius. This upper limit is largest for $u=1$. In this particular case, one finds $R \leq 1.21_{-0.28}^{+0.52} R_{\odot}$. This value, implausibly small for a B2 star, leads to question the validity of the model from which it is derived.

One possible shortcoming of this model might be that the actual field structure departs very significantly from a single dipole. However, the upper limit derived for the radius does not appear to depend critically on the field structure. For instance, the essentially sinusoidal variations of $\left\langle H_{z}\right\rangle$ observed in HD 96446 might be due to a quadrupolar field, in either of the following geometrical configurations: $i \approx 0$ (plausible as $v_{\mathrm{e}} \sin i \leq 16 \mathrm{~km} \mathrm{~s}^{-1}$ ), or $\beta \approx 90^{\circ}$ (then the actual rotation period of the star should be twice longer, i.e., 1.70$)$. In both cases, the same upper limit of the radius is derived: $R \leq 0.93_{-0.22}^{+0.40} R_{\odot}$.

Thus it appears that the variations of the magnetic field of HD 96446 cannot be satisfactorily explained by the ORM. Another mechanism should be sought. Pulsation is an obvious candidate, though it is at present unclear through which physical process it would induce variations of the longitudinal magnetic field. In relation to this, it is noteworthy that Matthews \& Bohlender $(1991)$ suspected the existence of a secondary periodicity $\left(P_{2} \approx 6.2\right)$ in the photometric variations of HD 96446, which they tentatively attributed to $\beta$ Cephei-type pulsation. This second periodicity is not obvious in the $\left\langle H_{z}\right\rangle$ data, but the latter are neither accurate enough nor sufficiently numerous to be quite conclusive in that respect.

The evidence presented in this poster does not question the general validity of the ORM for the interpretation of the variations of the majority of $\mathrm{Ap}$ and Bp stars (which are mostly cooler than HD 96446). But it calls for a closer investigation of the variations of HD 96446 as well as of other He-strong Bp stars. In particular, it may well turn out that these stars are not so closely related to the cooler Ap and Bp stars as is generally believed. More observations (both of the magnetic field and of photometric variations) are needed to settle this issue.

\section{References}

Matthews, J.M. and Bohlender, D.A.: 1991, Astron. Astrophys. 243, 148 